\title{
La remineralización de Europa: una aproximación
}

\author{
Juan Diego Pérez-Cebada* \\ María Jesús Beltrán Muñoz**
}

Resumen. La creciente demanda de minerales en el mundo y la situación de debilidad en los mercados internacionales de Europa han propiciado que la Unión Europea (UE) promueva distintas iniciativas, como la Raw Material Iniatiative (2008), claramente orientadas a fomentar la producción. A pesar de los enormes retos políticos, económicos, sociales y medioambientales que implica, la implementación de esa directiva sirvió de paraguas a una larga serie de «estrategias mineras nacionales» que han impulsado la «remineralización» del Viejo Continente. Ese proceso no fue uniforme, en especial después de la Gran Recesión, sino que las zonas de explotación se extendieron mayoritariamente por la periferia del continente. La intensa recuperación del sector dio lugar, además, a fuertes tensiones sociales. Es de particular interés en este contexto la investigación de la Faja Pirítica onubense y los conflictos derivados de la utilización del agua en la mina a cielo abierto Cobre Las Cruces, la más importante de Europa.

Palabras clave: Raw Material Initiative, periferialización, Cobre Las Cruces, conflictos mineros en Europa.

* Profesor titular del Departamento de Economía, Universidad de Huelva, España.

** Profesora ayudante en la Universidad Pablo de Olavide de Sevilla, España. 


\title{
The Remineralization of Europe: one approach
}

\begin{abstract}
The growing global demand for minerals and the weakness of international markets in Europe have pushed the European Union (EU) to promote various initiatives, such as the Raw Material Initiative (2008), clearly oriented toward driving production. Despite the enormous political, economic, social and environmental challenges it represents, the implementation of this policy will serve as an umbrella for a larger series of «national mining strategies» that have been employed toward the «remineralization» of the Old Continent. However, this process — particularly following the Great Recession - has not been uniform, as the areas of operation are located primarily on the periphery of the continent. The concentrated move to promote this sector also gave rise to significant social tension. Of particular interest in this context is research on the Andalucian Pyrite Belt and the conflicts arising from the use of water in the Cobre Las Cruces open-pit mine, the largest in Europe.
\end{abstract}

Keywords: Raw Material Initiative, peripherialization, Cobre Las Cruces, mining conflicts in Europe. 


\section{Introducción}

La expansión internacional de la minería es un componente esencial de la globalización económica tanto como los conflictos ambientales derivados de esas actividades (Bridge, 2004). El intenso crecimiento económico, la alta demanda de materias primas o las políticas neoliberales fueron los factores de fondo del boom minero de la década de 1990. En este mercado global, la posición de Europa, que consumía 25-30\% de los metales del mundo (aunque sólo produce 3\%) era débil. En ese contexto se van a promover distintas iniciativas nacionales con el objetivo de reactivar el sector minero-metalúrgico en el Viejo Continente, iniciativas que van a recibir un definitivo impulso a raíz del súper ciclo minero de 2003-2008, cuando la Unión Europea va a tratar de implementar una política minera común (Tiess, 2010; Herber, 2008). Sin embargo, la complejidad derivada de la aplicación de los principios de sostenibilidad implicó enormes desafíos sociales, institucionales o científico-técnicos (Endle, 2017). En paralelo, este proceso generó fuertes tensiones sociales en las cuencas, sobre todo después de que la Gran Recesión acelerara un proceso de remineralización que afectará especialmente a países periféricos (y dentro de ellos a regiones periféricas) con graves problemas económicos y donde, muy pronto, se multiplicarán los conflictos ambientales. ${ }^{1}$

En este artículo se pretende realizar una primera aproximación a esta cuestión. Para ello se aborda en el primer apartado el contexto en el que

${ }^{1}$ En 2016 se reunían en la Universidad de Évora (Portugal) un grupo de investigadores españoles, portugueses y brasileños para abordar los retos socioeconómicos y ambientales de la nueva minería en Europa. El resultado fue la publicación de un libro (Guimaraes-Pérez, 2016) en el que se plantea esa nueva línea de investigación. 
surge el proceso de remineralización del continente. El resurgir de la minería en los países de la periferia europea va a coincidir con movimientos de resistencia antiminera que son estudiados en el segundo apartado. Finalmente, en el tercer epígrafe se analiza con detenimiento uno de esos casos, el que afecta a la mayor mina de cobre a cielo abierto de Europa, Cobre-Las Cruces, en la Faja Pirítica Ibérica.

\section{El contexto de la reactivación minera en Europa}

El debate actual sobre el carácter «crítico» de los recursos mineros (y, en general, de las materias primas estratégicas) y sus implicaciones ambientales tiene profundas raíces históricas (Hauman, 2018). Es significativo el hecho de que, al menos desde finales del siglo XIX, la controversia sobre el agotamiento de los recursos mineros coincida con los problemas de degradación ambiental que causan las actividades extractivas. Ya entonces, en un contexto de acusada internacionalización de los circuitos comerciales, en las grandes cuencas del mundo se asiste simultáneamente a un fenómeno que había surgido en la primera mitad del siglo XIX en Europa: la multiplicación de conflictos sociales relacionados con la contaminación del aire, del suelo, de las aguas o de la biota. Conflictos que enfrentan a las grandes corporaciones mineras con los afectados por esas actividades en su salud o en sus actividades económicas (Pérez, 2014).

Un siglo después, una segunda oleada globalizadora va a coincidir con una fuerte expansión del sector minero apoyado en el denominado Consenso de Washington. De nuevo, las cuencas funcionan como un laboratorio de ensayo de la globalización con graves efectos socioambientales que 
afectan sobre todo al Sur Global, como se mostrará en otros artículos de este monográfico. La posición que Europa había mantenido en el corazón del sistema capitalista se había basado precisamente en la masiva importación de materias primas minerales procedentes de esa zona. ${ }^{2}$ Sin embargo, los estragos de la crisis de finales de los 1990 en el comercio internacional, la aparición de nuevos actores internacionales y su impacto sobre la demanda de minerales estratégicos hacen saltar las alarmas (Endle, 2017). Se suscita una grave preocupación entre las autoridades comunitarias sobre el carácter «crítico» de las materias primas, y muy especialmente de las mineras, para la economía europea. Con el fin de reducir la dependencia exterior y promover la oferta, la Comisión Europea elabora la Raw Materials Initiative (RMI, 2008) que, en consecuencia, va a tener inicialmente un doble componente económico y geopolítico. En cierto sentido, esa tendencia productivista tenía sus raíces en el propio acervo legal comunitario y en los tratados fundacionales. Para algunos investigadores, de ahí se derivaría una tradicional laxitud de las normativas medioambientales con el sector extractivo (Hamor, 2004; Amezaga et al., 2011; Šolar-Shields, 2006).

Por otro lado, es el momento también en el que las instituciones europeas adoptan los principios del Desarrollo Sostenible contenidas en el informe Brundtland. En el sector minero, el impacto causado por la rotura de las balsas de decantación de Aznalcóllar (España, 1998) y Baia Mare (Rumanía, 2000) aceleran el proceso al poner en evidencia la inadecuación de la normativa ambiental minera. Como consecuencia, dos comunicaciones (COM, 2000) y diversos documentos fueron publicados, hecho que instó a la actualización de esa normativa (Hamor, 2004).

${ }^{2}$ Desde los 1970 hasta el año 2000 se habían establecido acuerdos de cooperación para este fin con diversos países de África, el Caribe y el Pacífico (Tiess, 2010). 
En realidad, la inicial noción de «carácter crítico», aplicada a los recursos naturales mineros, ha evolucionado hacia una acepción más amplia que englobara las dimensiones medioambientales o sociopolíticas (Graedel, 2012; Mancini et al., 2018). La utilización de metodologías más inclusivas en esta línea ha sido abordada en diversos trabajos (Erdman-Graedel, 2011; Sonnemann et al., 2015). Recientemente, se ha abierto paso a una crítica más profunda, dirigida sobre todo al concepto que sustenta la RMI y las políticas ambientales de la Unión Europea (UE), la economía circular, partiendo de la idea de que la complejidad de los problemas afrontados obliga a una perspectiva multi e intradisciplinar. En síntesis, estos investigadores defienden que es la Ecología Política el único campo de conocimiento que puede ayudar a superar las grandes limitaciones de orden ecológico, social, político y cultural del discurso de la circularidad (Friant-Vermeulen-Salomone, 2019).

\section{Remineralización, periferialización y conflictos ambientales}

En todo caso, la RMI va a servir como documento guía que facilite la activación de «estrategias integradas» de los países europeos que permita hacer compatibles el incremento de la producción y la reducción de la dependencia europea con el Desarrollo Sostenible (Endl, 2017:3; Rayner-Howlet, 2009). Hay que tener también en consideración que el súper ciclo minero (2003-2008) había empujado a los distintos actores en el mercado internacional (Estados Unidos, Canadá, Australia, los BRICs, etcétera) a aprobar legislaciones que promovían el desarrollo minero nacional (Humphreys, 2010). En ese contexto, la UE jugaba, otra vez, en desventaja. 
Por tal razón, la función fundamental de esta directiva ha sido la de promover normativas nacionales inspiradas en ella, pues no hay que olvidar que el sector extractivo europeo sigue estando sujeto a la legislación minera de cada país (Hator, 2004; Tiess, 2010).

A resultas de esa directiva, entre 2010 y 2013 se van a activar políticas mineras en una decena de países europeos, a las que habría que sumar alguna legislación regional más tardía (como la de Andalucía en 2016). Es interesante constatar que siete de esos países promueven «Estrategias Mineras» (ocho, si incluimos a Andalucía) en sentido estricto, como delata su propia denominación. Los tres restantes extienden su rango a todos los recursos críticos («Resource Security Plan», Gran Bretaña; «Raw Material Strategy», Alemania) o los limitan a los recursos ligados a sectores como el automovilístico o el aeronáutico («Enjeux economiques des metaux strategics pour les filiéres automobile et aéronautique», Francia) o a la minería en el mar («Seabed Mining Act», Alemania). ${ }^{3}$

De los primeros, seis (o siete si tenemos en cuenta Andalucía) se sitúan en la periferia europea. Es verdad que los distritos de mayor potencial minero en el continente se encuentran en el arco que se dibuja desde la península ibérica a la península escandinava, pasando por los países del este y la península balcánica (Nurmi-Molnar, 2014). Sin embargo, más que a un cierto determinismo geológico, la periferialización de la minería europea parece responder, según algunos investigadores, a los procesos de desindustrialización, desempleo y emigración que experimentaron algunos de estos países (y dentro de ellos, algunas de estas cuencas) especialmente tras la Gran Recesión (Becker y Weissenbacher, 2015).

${ }^{3}$ Sustainable Supply of Raw Materials from EU Sources (http://ec.europa.eu/growth/sectors/ raw-materials/policy-strategy/sustainable-supply-eu/index_en.htm). 
Es sintomático también que la reactivación minera viniera acompañada de graves conflictos ambientales. Aunque la investigación está aún en un estado preliminar, ya hay trabajos académicos, algunos de ellos con una perspectiva comparativa, ${ }^{4}$ que permiten una primera aproximación al fenómeno. Sin ánimo de ser exhaustivo, se señalan a continuación determinadas líneas de investigación de interés. Se conoce bien, para empezar, que ya en los 1990 los países del Este se habían convertido en una nueva frontera de los recursos minerales, especialmente del oro, y se habían hecho atractivos para el sector extractivo internacional. Este es el caso especialmente de Rumania, que en 2013 representaba el modelo ideal para aplicar las «políticas mineras» entonces de moda en el resto de Europa (Marinescu-Kriz-Tiess, 2013). Es significativo que dos de los megaproyectos estrella de los 1990 deriven, ya en el nuevo siglo, en graves problemas medioambientales: el aludido de Baia Mare, cuyo vertido al Danubio escandalizó a Europa; y Roşia Montană, un histórico yacimiento que logró ser frenado gracias, entre otras razones, a las estrategias multiescalares de los movimientos de resistencia locales (Alexandrescu, 2013; Veliku-Kaika, 2015; Stefănescu-Robu-Ozonu, 2013).

En la primera década del siglo XXI se asiste también a un boom minero que se extiende desde el norte de Escandinavia al noroeste de Rusia y que coincide con múltiples movimientos de resistencia. Es destacable especialmente el caso de la mayor potencia minera de la UE, Suecia, que se ha convertido de igual modo en un ejemplo internacional de «buenas prácticas» ambientales en un sector que, sin embargo, se enfrenta a una fuerte oposición. Las demandas vinculadas a la justicia ambiental, características de otros

${ }^{4}$ Véase, por ejemplo, Landén-Fotaki, 2018; Lesser et al., 2016; Suopajärvi et al., 2017; FloreaRhoades, 2019. 
conflictos, se entrecruzan en este país, como en todo el norte de Escandinavia, con las reivindicaciones de los criadores de renos sami cuyo frágil entorno se ve radicalmente modificado por las actividades extractivas y con la denuncia de que las cuencas mineras funcionaban como «colonias internas», en las que un desarrollado sur explota a un depauperado norte (Haikola-Anshelm, 2016; Landén-Fotaki, 2018; Lesser et al., 2016; Suopajärvi et al., 2016).

También en la península balcánica se ha combinado un acusado proceso de reactivación minera con movimientos de protesta anticontaminación en las dos últimas décadas. Es sin duda el caso griego el que más ha contribuido a cimentar la idea de que la Gran Recesión ha sido la excusa para promover políticas de austeridad de corte neoliberal en el sector con graves consecuencias medioambientales, como muestra el espectacular caso de las históricas minas de oro de la península de Halkidiki. En 2012 se hacía con los derechos de estos yacimientos una gran corporación minera canadiense, ELdorado Gold. La irrupción de un activo y sostenido movimiento de protesta antiminero, con una sobresaliente presencia femenina en su liderazgo, y la oposición del gobierno de Syriza lograron detener este proyecto. No obstante, el reciente gobierno de Nueva Democracia concedió en 2018 los permisos correspondientes a la empresa para reiniciar los trabajos de extracción (Calvário-Velegrakis-Kaika, 2016; Velegrakis, 2016; Landén-Fotaki, 2018).

Como se adelantaba, en 1998 la rotura de la balsa de Aznalcóllar ${ }^{5}$ iba a poner en evidencia los enormes riesgos ambientales de la nueva minería en Europa. El súper ciclo minero y la Gran Recesión favorecieron el renacimiento minero de España y Portugal, ambos países con un largo historial de problemas de contaminación en este ámbito. Los conflictos

${ }^{5}$ La rotura de la presa de estériles mineros que tuvo lugar en abril de 1998 en esa explotación ha supuesto el accidente medioambiental más severo acontecido en España, no sólo por el vertido 
ambientales se dispararon desde entonces afectando a una buena parte de las cuencas tradicionales pero también a los nuevos yacimientos, especialmente de tierras raras. ${ }^{6}$ La diversidad y gravedad de esos casos ha llevado a plantear la sugerente hipótesis de que la minería peninsular, y más específicamente la que se practica en la Faja Pirítica Ibérica, ha servido como un auténtico «laboratorio» de la minería continental (Koven, 2012). En ese sentido, cobra un interés especial el caso de Cobre Las Cruces, que se aborda con extensión a continuación.

\section{Cobre Las Cruces}

El proyecto Cobre Las Cruces resulta un caso significativo en el contexto de la remineralización de Europa y los conflictos ambientales que ha desencadenado por dos razones principales: por un lado, es la explotación minera que mayor producción de cobre aporta al sector de la minería en España. Por el otro, el análisis que se presenta del caso ejemplifica un conflicto ambiental en torno al uso del agua, ya que muestra las estrategias que la compañía minera ha aplicado para apropiarse del recurso hídrico, imprescindible para el proyecto. En este caso, el movimiento de resistencia ante los impactos producidos por la explotación se ha ejercido principalmente mediante acciones legales, emprendidas por un grupo ecologista

de $6.8 \mathrm{hm}^{3}$ a un río cercano, sino por la presencia del Parque Nacional de Doñana aguas abajo donde se produjo el accidente. Para más información véase en este monográfico el artículo de Félix Talego y Maika Zampier.

${ }^{6}$ Algunos de estos casos se abordaron en el libro editado por Guimaraes y Pérez (2016). Una aproximación más sistemática, desde el lado portugués, es el capítulo «Mineraçao» del libro de Chaves-Oliveira (2019:263-320), que se centra en el análisis de varios casos destacados. 
local (Ecologistas en Acción), que ha llevado entre otros casos a la condena contra tres directivos de CLC por delitos medioambientales.

En primer lugar, la importancia de Cobre Las Cruces en el nivel monetario en la economía española viene caracterizada por las estadísticas del sector minero: Cobre Las Cruces es la explotación que más producción de cobre aportó al subsector de la minería metálica en España, siendo éste el que a su vez más valor monetario aportó al sector minero en su totalidad en el año 2016 con más de 700 millones de euros, con sólo nueve explotaciones en activo en todo el país (Instituto Geológico y Minero de España, IGME, 2017). De entre todas las Comunidades Autónomas, cabe destacar que Andalucía es la región que más valor de la producción aportó al subsector de la minería metálica. Las estadísticas de la extracción de metales, muestran a su vez que la producción de cobre es, de entre todos los metales, el que ha experimentado mayor crecimiento, con incremento de $2400 \%$ de 2008 a 2016 (IGME, 2017). La extracción de cobre metálico está localizada en las provincias de Huelva, con una producción de 602 kt en 2016, y Sevilla, que duplicó esta cantidad con una producción de 1180 kt en el mismo año. Esta extracción de cobre se realiza en la única explotación en activo localizada en la provincia de Sevilla, Cobre Las Cruces (IGME, 2017).

Para esta destacada producción de cobre el agua es un recurso fundamental. Así, el acceso a los recursos extractivos depende tanto de la tecnología y de los precios como del acceso y control de los recursos hídricos, imprescindibles para las explotaciones mineras (Beltrán y Velázquez, 2017). Uno de los principales impactos ambientales que produce la minería metálica es el uso y contaminación de agua, ya que estos procesos necesitan elevados volúmenes de agua; asimismo, generan gran cantidad de aguas ácidas altamente contaminantes. Estos impactos ambientales 
permanecen ocultos en muchos casos si se analizan desde la red analítica de la economía convencional, centrada fundamentalmente en el estudio de los valores monetarios. En este caso para poner de manifiesto los impactos ambientales que supone la actividad minera del proyecto Las Cruces vamos a centrar la atención en analizar el papel de las instituciones, entendidas como «reglas del juego» (Cárdenas y Ostrom, 2004, 309), que condicionan la actual gestión del agua que se lleva a cabo en el proyecto.

El proyecto minero Las Cruces (CLC), como se aprecia en la figura l, se sitúa al sur de España, en la Comunidad Autónoma Andaluza, concretamente en el cuadrante noroccidental de la provincia de Sevilla, ocupa 950 hectáreas en los términos municipales de Gerena, Guillena y Salteras, a 15 kilómetros de la capital sevillana. Pertenece a la empresa Cobre Las Cruces S.A., filial de la empresa canadiense First Quantum Minerals. Este proyecto minero destaca por la particularidad que supone la gestión del agua. El yacimiento de la mina Las Cruces se encuentra por debajo de un acuífero y para poder explotarlo la compañía diseñó un sistema conocido como Sistema Drenaje Reinyección (SDR) que proponía drenar el agua del acuífero únicamente en el área donde se localiza el yacimiento, como más adelante se explica. 
Figura 1

Localización de la explotación minera Las Cruces

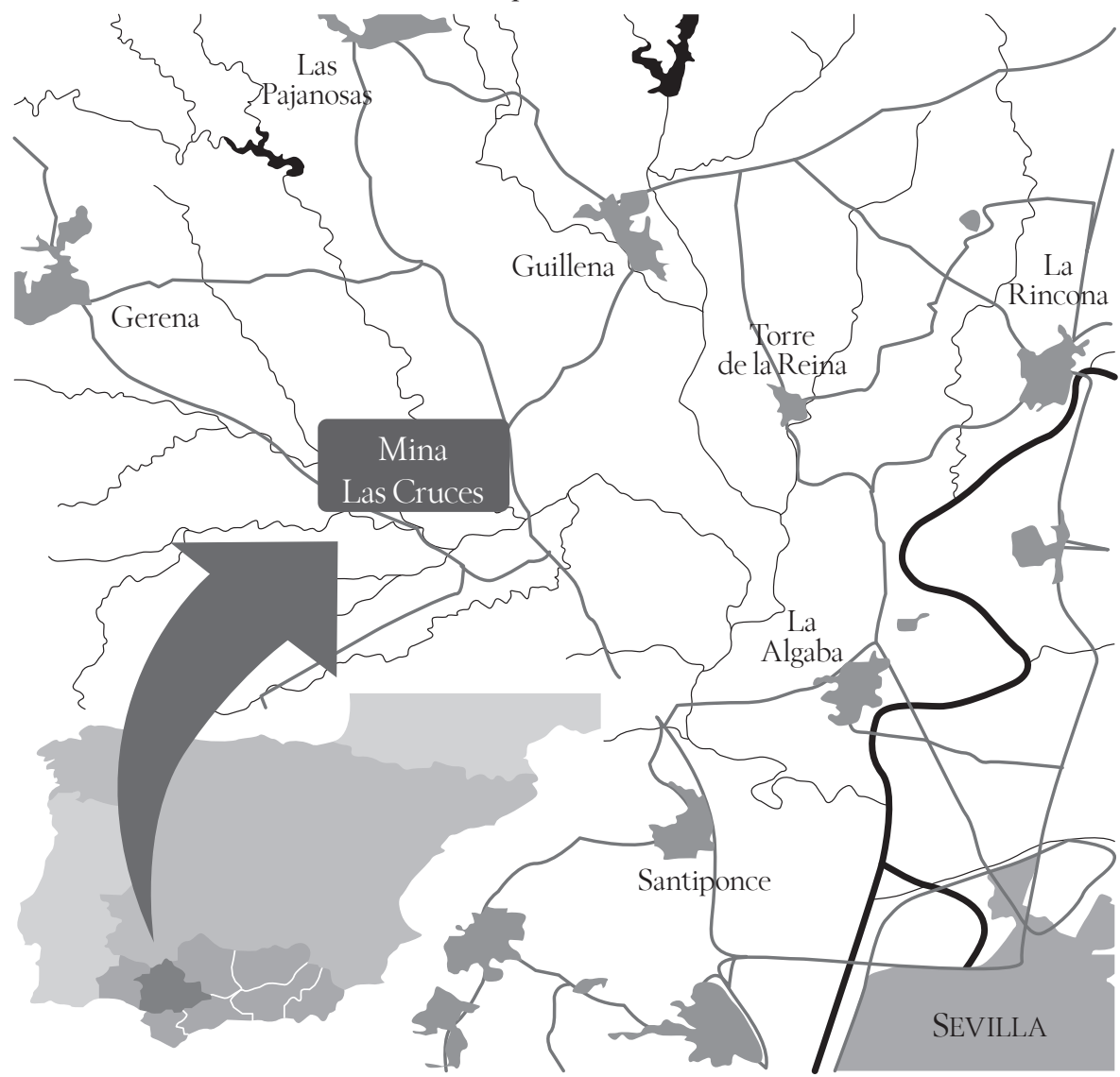

Fuente: Agencia Andaluza del Agua (2009).

Este sistema fue autorizado por la autoridad competente en materia de aguas, la Confederación Hidrográfica del Guadalquivir (CHG) en $2003^{7}$

${ }^{7}$ Autorización de las obras para la ejecución de instalaciones y actividades de operación de drenaje-inyección, en los términos municipales de Gerena, Guillena y Salteras (Sevilla) del 30 de octubre de 2003. 
con la condición de que no afectara a la calidad ni a la cantidad del agua del acuífero en cuestión (CHG, 2003). No obstante, en mayo de 2008, unos meses después de la puesta en marcha del SDR, la CHG decretó la suspensión del sistema debido a incumplimientos de la autorización, que provocaron a su vez la paralización de la actividad minera por parte de la Junta de Andalucía. ${ }^{8}$ Para poder retomar la actividad, la empresa presentó un nuevo Plan Global de Gestión de Aguas que fue aprobado en 2009 por la Junta de Andalucía. ${ }^{9}$ Este apartado presenta un análisis del Plan que permite examinar el papel que han desempeñado las instituciones en la gestión del agua del proyecto y que revela cómo las reglas del juego que condicionan dicha gestión han beneficiado intereses privados a expensas de intereses sociales y ambientales.

\section{El proyecto minero y la gestión de agua}

El área del proyecto se sitúa entre las unidades geoestructurales de la depresión del Guadalquivir y el extremo más oriental de la Faja Pirítica Ibérica que se caracteriza por la existencia de numerosos yacimientos de sulfuros polimetálicos y pirita como el de río Tinto (Huelva) o el de Los Frailes (Aznalcóllar, Sevilla). El yacimiento Las Cruces se encuentra a una profundidad de 150 metros, bajo 20 metros de areniscas y conglomerados

\footnotetext{
${ }^{8}$ Resolución del 14 de mayo de 2008 de la Consejería de Innovación, Ciencia y Empresa por la que se suspenden provisionalmente los trabajos de profundización de la corta, en la explotación de recursos de la sección C denominada Las Cruces, número de registro minero 7532-A, cuyo titular es la entidad mercantil Cobre Las Cruces S.A.

${ }^{9}$ Resolución de autorización de modificación de características de las obras consistentes en la ejecución de instalaciones y actividades de operación de drenaje-inyección, en los términos municipales de Gerena, Guillena y Salteras (Sevilla) del 16 de julio de 2009.
} 
del acuífero Niebla-Posadas. Las reservas de cobre constituyen el fundamento del proyecto minero: se estiman en 16 millones de toneladas con una ley de 6.2\% en cobre; esto quiere decir que cada tonelada de mineral removido contiene una media de $62 \mathrm{~kg}$ de cobre. Esta característica hace que el yacimiento se considere muy rentable desde el punto de vista monetario.

La actividad extractiva del proyecto minero comprende la extracción del mineral del yacimiento en mina a cielo abierto (corta minera), seguida de la extracción del metal (cobre) del mineral en la planta hidrometalúrgica. En la planta los materiales extraídos se someten a enriquecimiento por lixiviación y extracción con disolventes, para poder obtener el cobre metálico en un proceso de electrodeposición, en el que se consigue cobre metal de alta pureza en forma de láminas de cobre (también llamados cátodos de cobre) (FRASA y AIA Consult, 2005). Tanto de la corta minera como de la planta surgen rocas estériles que contienen minerales que deben ser tratados debido a su elevado riesgo de contaminación. El tratamiento de esos estériles se realiza mediante deposición en seco. Ello significa que los estériles en primer lugar se filtran para reducir el grado de humedad y acto seguido se encapsulan mediante geomembranas y margas impermeables que aseguran el sellado de los materiales. Dicho método se considera ventajoso frente al depósito tradicional en balsa de lodos al minimizar el riesgo de contaminación por vertido de aguas ácidas y supone una destacada diferenciación del proyecto minero de Aznalcóllar ya mencionado.

El agua es un elemento indispensable para el tratamiento hidrometalúrgico y debe ser gestionada en todo el proceso minero. Así, el proyecto comprende acciones con respecto a las aguas subterráneas y superficiales. Las fuentes de agua disponibles para el suministro a la planta autorizadas 
por la CHG mediante una concesión aprobada en el año $2004{ }^{10}$ corresponden a $296.8 \mathrm{~m}^{3}$ /hora/año (2.6 millones de $\mathrm{m}^{3}$ /año) de agua residual urbana depurada que la Estación Depuradora de Aguas Residuales (EDAR) de San Jerónimo (situada al norte de Sevilla) vierte al río Guadalquivir (este volumen supone 10\% del caudal de agua depurada que la EDAR vierte al río); junto con un volumen de $18.3 \mathrm{~m}^{3} /$ hora/año (160 mil m³/año) de aguas remanentes de la explotación minera (aguas de contacto del fondo de la corta).

La principal actuación con relación a la gestión de agua subterránea es la puesta en marcha del SDR en la que se determinaba como condición la no afección a la calidad de las aguas del acuífero y la no detracción de agua del mismo. Como se ha comentado anteriormente, el yacimiento de la mina Las Cruces se encuentra sumergido bajo el agua de un acuífero y para poder explotarlo el SDR se diseñó con intención de drenar el agua del acuífero únicamente en el área donde se localiza el yacimiento. Esto se consigue mediante una serie de sondeos de drenaje que extraen el agua del acuífero conectados a su vez a unos sondeos de inyección que retornan el agua al acuífero fuera de la zona donde se sitúa el yacimiento (figura 2). El objetivo del SDR era que el agua del acuífero no entre en contacto con los minerales de la corta minera. Así, este modelo debía garantizar que toda el agua que se extraiga del acuífero en la zona de corta fuese retornada para mantener la cantidad y la calidad de éste.

En la figura 2 se expone un esquema del funcionamiento del SDR en la corta minera, en el que se observa cómo el nivel del agua del acuífero se deprime en los sondeos de extracción y cómo se eleva en los sondeos de

${ }^{10}$ Concesión de Aguas Públicas TC 17/2017 del 17 de junio de 2004. 
reinyección. El acuífero se compone de areniscas del Mioceno de base y el yacimiento se encuentra en las rocas paleozoicas, aproximadamente, bajo el relleno de margas.

\section{Figura 2}

Esquema del Sistema Drenaje Reinyección del complejo minero Las Cruces

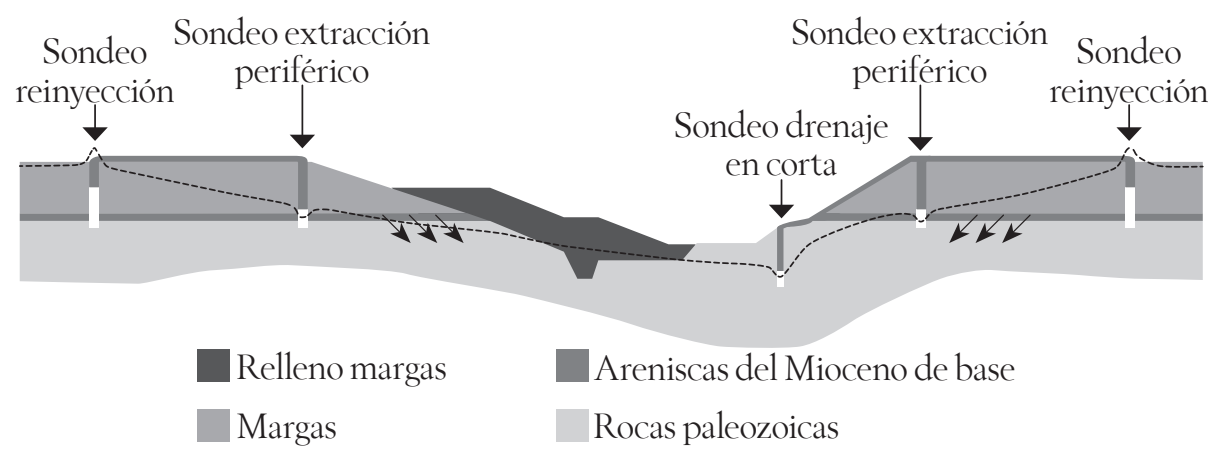

Fuente: Cobre Las Cruces (2008).

\section{Contexto institucional que condiciona la actual gestión}

de agua del proyecto Las Cruces. ¿A quiénes benefician

y a quiénes perjudican las reglas del juego?

Con anterioridad se comentó que la gestión actual del proyecto minero se basa en el Plan Global de Gestión de Aguas. A modo de introducción, en 2003 la CHG otorgó a Cobre Las Cruces la autorización para el SDR, en la que se determinaba como condición la no afección a la calidad de las aguas del acuífero y la no detracción de agua del mismo. No obstante, en mayo de 2008, la CHG decretó la suspensión del SDR del proyecto minero 
Las Cruces, por graves y reiterados incumplimientos de las condiciones impuestas en la autorización del SDR de 2003, ya que durante la puesta en marcha del sistema se comprobó la detracción de aguas de contacto no compatibles con los términos de lo autorizado. ${ }^{11}$ Al día siguiente, la Junta de Andalucía decretó la paralización de las labores de profundización en corta y afloramiento del mineral (Junta de Andalucía, 2008), situación que corroboró técnica y administrativamente que la mina no puede funcionar sin el SDR. En septiembre de 2008 la empresa presentó a la CHG el Plan Global de Gestión de Aguas para levantar la suspensión impuesta al SDR (Cobre Las Cruces, 2008). Con ello modificó el SDR y fue aprobado por la Junta de Andalucía en julio de 2009, lo que permitió el levantamiento a la suspensión impuesta, así como la aprobación del Plan Global de Gestión de Aguas. El Plan supuso a su vez la contratación de la figura del hidrogeólogo residente, un experto de la CHG que pertenece al personal de la compañía minera y que remite informes cuantitativos y cualitativos del estado de las aguas superficiales y subterráneas en el área de la explotación minera a la CHG.

Los incumplimientos que se detectaron derivaban, entre otros aspectos, de que el modelo hidrogeológico y de gestión implementado por Cobre Las Cruces contenía importantes modificaciones con respecto al que sirvió de base para la autorización y la empresa comenzó su actividad sin informar de la modificación citada. Con relación a la cantidad de agua, el modelo autorizado en 2003 se basaba en la necesidad de reintegrar lo extraído al acuífero. Esto es, no se permitían las detracciones netas de agua del acuífero. Sin embargo, se confirmó la detracción no autorizada de 75

${ }^{11}$ Cobre Las Cruces. Propuesta de suspensión del 30 de abril de 2008 de la Autorización de drenaje reinyección del 30 de octubre de 2003. 
mil $\mathrm{m}^{3}$ de agua del acuífero Niebla-Posadas y la práctica de 20 sondeos no autorizados. Concerniente a la calidad, se detectó el vertido de arsénico y otras sustancias contaminantes a este cauce hídrico. ${ }^{12}$

El Plan Global de Gestión de Aguas supone una modificación de los volúmenes de agua estipulados en la concesión de 2004, pues el modelo hidrogeológico propuesto contempla el uso de $157.83 \mathrm{~m}^{3} /$ hora/año de aguas remanentes de la explotación minera, lo que supone 876\% más de lo estipulado en la concesión autorizada. Esta agua, calificada también como «agua de mina» ${ }^{13}$ según el Plan, se incorpora como agua de proceso para el tratamiento hidrometalúrgico, previo tratamiento de ósmosis inversa, minimizando así el uso del agua proveniente de la EDAR de San Jerónimo. Lo anterior supone una reducción del volumen que extrae la tubería de abastecimiento del río Guadalquivir, en detrimento del aumento de volumen de agua de mina. Otra modificación destacable que propone el Plan consiste en que para garantizar la calidad de las aguas del acuífero se tratan las aguas del SDR que entran en contacto con el mineral mediante ósmosis inversa. Como resultado del proceso de ósmosis inversa, una parte de la cantidad de agua tratada no se puede volver a reinyectar en el acuífero. Esto significa que $54 \mathrm{~m}^{3} / \mathrm{hora} /$ año de las aguas del acuífero detraídas del acuífero no se reinyectan, lo que constituye una contradicción a las normas de explotación del acuífero que prohibían las detracciones netas de agua.

${ }^{12}$ Resolución de autorización de modificación de características de las obras consistentes en la ejecución de instalaciones y actividades de operación de drenaje-inyección, en los términos municipales de Gerena, Guillena y Salteras (Sevilla) del 24 de octubre de 2013.

${ }^{13}$ Instituto Geológico y Minero de España (IGME) (2010): Informe sobre la solicitud del Plan Global de Cobre Las Cruces y levantamiento de la suspensión de la Autorización de Drenaje-Inyección, del 15 de abril de 2010 para el procedimiento de Diligencias previas 7176/2008 del Ministerio Fiscal contra Cobre Las Cruces. 
En ese sentido, en la cláusula cuarta de la autorización otorgada al Plan se propone iniciar el procedimiento de modificación de la concesión de 2004. Dicha modificación es, en teoría, imprescindible para poder alterar los volúmenes de uso de agua en la explotación (concretamente, para ampliar la utilización de aguas remanentes de la mina o aguas de mina y detraer la cantidad de agua propuesta mediante el SDR). Llegados a este punto, cabe preguntarse: ¿cómo fue puesto en marcha un plan de gestión de aguas que permitía la modificación de los volúmenes usados de recursos hídricos sin la previa modificación obligatoria de la concesión de aguas, y que a su vez afectaba a un acuífero en el que las normas de explotación no permitían detracciones netas de agua? Tal pregunta señala dos aspectos clave del caso que a continuación se desarrollan: el papel del discurso científico para legitimar el uso indiscriminado de agua subterránea por parte de la compañía minera, y la importancia de las reglas del juego para permitir a la compañía minera apropiarse del recurso hídrico.

En primer lugar, en lo que respecta a la afectación del acuífero, la autorización otorgada al Plan Global de Gestión de Aguas expone que el volumen de agua considerada como agua de mina no procede del acuífero Niebla-Posadas, puesto que según el estudio hidrogeológico realizado sobre el acuífero por el IGME en 2010 el agua que lo constituye se encuentra sólo en las areniscas del Mioceno de base. El agua de mina proviene del Paleozoico (véase figura 5), que es el material donde se encuentra el yacimiento. Ello supone que el agua surge «de forma natural» a medida que se profundice la corta (Cobre Las Cruces, 2008). Paradójicamente, en el mismo Plan se describe el acuífero, el cual indica que ambos materiales, Paleozoico y areniscas, se encuentran fracturados y «pueden entenderse como parte del mismo acuífero» (Cobre Las Cruces, 2000:14), por 
lo que contradictoriamente a lo que indica el informe geológico del IGME, el agua de mina está en contacto con el agua del acuífero, es decir, no hay diferencia entre agua de mina y agua del acuífero (Ramos, s/f). Este dato, puesto de manifiesto en un informe técnico elaborado por Ecologistas en Acción, revela un aspecto clave: la introducción del término científico de agua de mina ha sido usada para legitimar el aumento de $876 \%$ de volumen de agua procedente del acuífero. De esa forma, habría que cuestionar si la utilización de ese concepto científico (sujeto a la incertidumbre en el conocimiento de la dinámica del acuífero) está silenciando los impactos provocados por un sistema que tenía como objetivo proteger el acuífero.

Dicha controversia se ve reflejada en los datos de los informes trimestrales del hidrogeólogo residente (2015-2019) acerca del estado cuantitativo del acuífero. En esos informes se mide el nivel freático de cinco sondeos localizados en la explotación y se compara dicho nivel con el nivel que debería tener según las previsiones del Plan Global de Gestión de Aguas. Por ejemplo, el punto del gráfico del sondeo de Torre de la Reina para el año 2015 muestra que dicho sondeo está 18 metros por debajo de la previsión. Así, en el gráfico, la evolución positiva muestra un peor estado cuantitativo del acuífero en la zona de ese sondeo. El resultado de la evolución se muestra en la figura 3. Todos los sondeos, con especial atención al denominado Render Grasas, se encuentran por debajo del nivel piezométrico que se preveía en el Plan Global de Gestión de Aguas. Tales datos muestran el impacto cuantitativo que está sufriendo el acuífero en el área del proyecto. En ese sentido, en marzo de 2018 la CHG formuló una propuesta para una sanción de un millón de euros y una indemnización de 496 mil 882.94 euros por detracción ilegal de aguas subterráneas entre el 12 de febrero de 2015 y octubre de 2016 por infracción muy grave cometida por la empresa 
Cobre Las Cruces, que elevó al Consejo de Ministros para su resolución definitiva y está pendiente de resolución (CHG, 2018).

\section{Figura 3}

Evolución del nivel piezométrico de los sondeos localizados en el acuífero en el área de explotación del proyecto minero Las Cruces. Metros por debajo de la previsión del Plan Global de Gestión de Aguas, 2015-2019

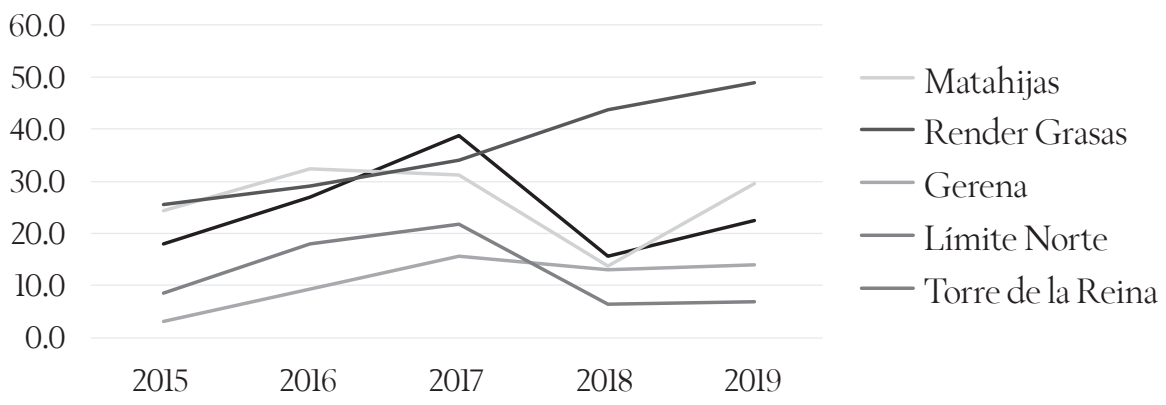

Fuente: informe del hidrogeólogo residente para la Confederación Hidrográfica del Guadalquivir (2015-2019).

En segundo lugar, hasta la aprobación del actual Plan 2015-2021 de la Demarcación Hidrológica del Guadalquivir en 2016, ${ }^{14}$ se estuvo realizando el proceso de modificación de la concesión de aguas otorgada a la minera en 2004 de forma paralela al trámite de declaración de sobreexplotación del acuífero que comenzó en diciembre de 2008. Aunque el plan de demarcación previo (2009-2015) consideraba la masa de agua subterránea

${ }^{14}$ Ministerio de Agricultura, Alimentación y Medio Ambiente (2016). Disposiciones normativas del Plan Hidrológico de la Demarcación Hidrográfica del Guadalquivir. BOE de 19 de enero de 2016. 
afectada por la explotación en riesgo de sobreexplotación por el mal estado cuantitativo, el plan actual ha dividido el acuífero en subunidades, de las cuales la que se ve afectada por la explotación minera es la denominada «Gerena» (Ministerio de Agricultura, Alimentación y Medio Ambiente, 2016). Paradójicamente la masa "Gerena» no se considera en mal estado cuantitativo, lo cual beneficia a la empresa minera al hacer factible la modificación de la concesión.

Paralelo al procedimiento administrativo que finalizó con la aprobación del Plan y la implantación de un nuevo modelo de gestión de aguas en la mina, se han desarrollado varios procedimientos judiciales contra Cobre Las Cruces, derivados, entre otros, de los recursos interpuestos por Ecologistas en Acción contra la aprobación del Plan Global de Gestión de Aguas de 2009. Como resultado, en septiembre de 2016 fueron declarados culpables y condenados a un año de prisión tres directivos de la mina Cobre Las Cruces por haber contaminado el acuífero Niebla-Posadas, además de pagar una multa de 6 mil 750 euros y condenar a la compañía a indemnizar al Estado con 293 mil 209.6 euros por los daños causados al dominio público (Europa Press, 2016).

Los procedimientos legales ya resueltos y los pendientes de resolución muestran que la empresa Cobre Las Cruces está siendo sancionada e investigada por detracción ilegal de aguas subterráneas, pero esa condición no ha impedido que el proyecto siga manteniendo sus perspectivas de producción de cobre y otros minerales. De hecho, la empresa está pendiente de los permisos de la Junta de Andalucía y la CHG para prolongar la explotación como mínimo hasta 2034 mediante un nuevo proyecto de explotación subterránea, que se construirá hasta 200 metros de profundidad bajo la corta actual (ABC de Sevilla, 2020). 


\section{Conclusiones}

En la última década se ha suscitado una comprensible preocupación en la opinión pública de las sociedades occidentales por las terribles consecuencias sociales y medioambientales de los conflictos mineros en determinadas zonas en conflicto en el Sur global. Tal concienciación llevó al presidente Barack Obama a decretar en 2010 la Dodd-Frank Act, que obligaba a las empresas mineras a informar sobre su participación y, en su caso, acerca de las medidas puestas en marcha para prevenir los conflictos mineros en la región de los Grandes Lagos, en África. Con similar filosofía, pero ampliando el radio de acción a todo el Sur global, en marzo de 2017 fue aprobada en el Parlamento Europeo la EU Mineral Regulation (Cuvelier, 2017). Esa loable inclinación de la opinión pública occidental había tenido un cierto correlato en el mundo académico, por cuanto la atención de los especialistas en el campo de la ecología política (y de la ecología política de la extracción) se ha centrado tradicionalmente en los conflictos que tenían lugar más allá del primer mundo y normalmente en el ámbito rural, mientras que han sido minoría aquellos que han analizado problemas de justicia ambiental en el mundo occidental, con frecuencia situados en un contexto urbano (Apostolopoulou y Cortés-Vázquez, 2019).

Sin embargo, en la última década la expansión de la gran minería ha provocado graves problemas ambientales en Europa a los que los investigadores están prestando cada vez más atención. En ese contexto, cabe plantearse varias consideraciones a modo de hipótesis, dado el estado preliminar de dichas investigaciones. La primera de esas consideraciones es que el inicial interés de la academia, en muchos casos inducido por los propios movimientos sociales, no ha derivado en un auténtico debate 
público sobre una cuestión tan relevante y, a su vez, tan compleja. Salvo en contadas ocasiones, el fenómeno parece haber estado invisibilizado. En consecuencia, se ha impuesto una política de hechos consumados que ha tenido como colofón las distintas legislaciones mineras nacionales a las que ha prestado cobertura la RMI (Tiess, 2010). Por otro lado, la propia geografía de las legislaciones es indicativa de una nueva reordenación espacial del trabajo en la que determinadas regiones periféricas se especializan en actividades mineras de bajo valor añadido, muy dependientes del exterior y del gran capital internacional. Es un tipo de extractivismo de tercera generación (Gudynas, 2017), muy tecnificado y con balances de flujos de materiales y energía muy ineficientes desde un punto de vista ecológico. En todo caso, como en otras zonas del Sur global antes, parece obvio que las fronteras del extractivismo se han extendido a Europa en la última década, en una fase avanzada de la globalización. En efecto, si el boom de las commodities impulsó el extractivismo minero en Latinoamérica, el impacto de la Gran Recesión parece haber sido decisivo en la activación a gran escala del sector en Europa: la UE, al reproducir la lógica de la «doctrina del shock» (Klein, 2007), desarrolló políticas de corte neoliberal que permitirán la definitiva implantación de las grandes corporaciones mineras en aquellas economías periféricas más vulnerables, como la española.

Desde un contexto micro y abordando uno de los más graves problemas de contaminación minera en la actualidad, el análisis de la gestión del agua del proyecto minero Las Cruces arroja también varias conclusiones de interés. En primer lugar, el concepto de agua de mina ha sido utilizado con el propósito de eliminar del discurso oficial el daño cuantitativo provocado al acuífero y evitar las objeciones normativas que surgen de la extracción de grandes volúmenes de agua subterránea para el proceso 
minero. La evolución que han sufrido las normas que rigen la caracterización y las normas de explotación del acuífero han allanado el camino hacia la legitimización de un plan de aguas que fue aprobado en circunstancias irregulares, puesto que no contaba con la modificación obligatoria de la concesión y afectaba a un acuífero considerado en mal estado cuantitativo. El análisis del marco institucional revela que es la combinación entre la mutación de las reglas del juego y el discurso científico (con la introducción del concepto de agua de mina) lo que hace posible legitimar una gestión de aguas que, como muestran los procedimientos judiciales aún abiertos, ha creado numerosas dudas concernientes a la legalidad de las actuaciones de la compañía minera. El papel ejercido en este caso por Ecologistas en Acción ha sido crucial con el fin de poner de manifiesto que las reglas del juego que rigen la gestión del agua en el proyecto minero Las Cruces han beneficiado intereses privados frente a intereses sociales y ambientales de protección del acuífero. Sólo el tiempo revelará los impactos que la sociedad tendrá que asumir derivados del daño al acuífero Niebla-Posadas, ahora silenciados gracias al concepto de «aguas de mina».

\section{Referencias}

ABC de Sevilla (6 de febrero de 2020). «First Quantum deja de explotar Cobre Las Cruces a la espera del permiso de la Junta para ampliar la mina». $A B C$ de Sevilla.

Agencia Andaluza del Agua (2009). «Minimización del volumen de vertido al

Dominio Público Marítimo Terrestre (río Guadalquivir) en el proyecto minero Las Cruces». En Comisión de Aguas. Consejo de Participación de 
Doñana Agencia Andaluza del Agrua. España: Agencia Andaluza del Agua. Recuperadodehttps:/www.ecologistasenaccion.org/wp-content/uploads/ adjuntos-spip/pdf_Informe_de_Comision_Agua_7_abril_2009.pdf

Alexandrescu, F.M. (2012). Human agency in the interstices of structure: choice and contingency in the conflict over Roşia Montană, Romania (tesis de doctorado). Canadá: University of Toronto.

Amezaga, J.M. et al. (2011). «A rich vein? Mining and the pursuit of sustainability». Environmental Science and Technology, 45, pp. 21-26.

Apostolopoulou, E. y Cortés-Vázquez, J.A. (2019). «Introduction. Neoliberalism and Environmental movements across the world after the 2008 financial crash: defending the right to nature». En Apostolopoulou, E. y Cortés-Vázquez, J.A. (eds.), The right to nature: social movements, environmental justice and neoliberal natures (pp. 1-13). Londres: Routledge.

Becker, J.J. y Weissenbacher, R. (2015). «Uneven and dependent development in Europe: the crisis and its implications». En Jäger, J. y Springler, E. (eds.), Asymmetric crisis in Europe and possible futures: critical political economy and post-keynesian perspectives (pp. 81-97). Londres: Routledge.

Beltrán, M. J. y Velázquez, E. (2017). «The political ecology of water metabolism. The case of the Cobre Las Cruces copper mine, southern Spain». Sustainability Science, 12(2), pp. 333-343.

Bridge, G. (2004). «Contested terrain: mining and the environment». Annual Review of Environment and Resources, 29, pp. 205-259.

Calvário, R., Velegrakis, G. y Kaika, M. (2016). «The political ecology of austerity. An analysis of socio-environmental conflict under Greece». Capitalism, Nature, Socialism, 28(3), pp. 69-87. 
Cárdenas, J. y Ostrom, E. (2004). «What do people bring into the game? Experiments in the field about cooperation in the commons». Agricultural Systems, 82, pp. 307-326.

Cobre Las Cruces (2008). Sistema de drenaje-reinyección del complejo minero-hidrometalúrgico Las Cruces. Síntesis del Plan Global de Gestión de Aguas. Recuperado de www.cobrelascruces.com

Confederación Hidrográfica del Guadalquivir (2018). Propuesta de resolución 213/17 SE. España: Gobierno de España.

Cuvelier, J. (2017). «Leaving the beaten track? The EU regulation on conflict minerals». Africa Policy Brief, 20, pp. 1-7.

Chaves, F.R. y Oliveira, L. (2019). Portugal: ambiente em movimento. Évora-Río de Janeiro: CEICP/CETEM.

Ecologistas en Acción (2008). Crónica de una catástrofe anunciada. Recuperado de www.ecologistasenaccion.es

Endle, A. (2017). «Addressing ‘wicked problems〉 through governance for sustainable development. A comparative analysis of national mineral policy approaches in the European Union». Sustainability, 9/10(1830), pp. 1-22.

Erdmann, L. y Graedel, T.E. (2011). «Criticality of non-fuel minerals: a review of major approaches and analyses». Environmental Science \& Technology, 45, pp. $7620-7630$.

Europa Press (2016). «Condenados a un año de cárcel y 6,750 euros de multa tres directivos de Cobre las Cruces». Recuperado de https://www.europapress. es/

Florea, I. y Rhoades, H. (2019). «Possibilities and pitfalls of environmental justice action. Learning from Roşia Montană and Yaigojé Apaporis anti-mining struggles». En Apostolopoulou, E. y Cortés-Vázquez, J.A. (eds.), The right to 
nature: social movements, environmental justice and neoliberal natures (pp. 84-97). Londres: Routledge.

FRASA y AIA Consult (2005). Cobre Las Cruces. Proyecto minero-hidrometalúrgico. Valoración ambiental del estado preoperacional. Sevilla: Cobre Las Cruces S.A.

Friant, M.C., Vermeulen, W. y Salomone, R. (2019). «Advancing a critical research agenda on the circular economy». En 25th International Sustainable Development Research Society Conference (pp. 46-61). Nanjing: ISDR.

Graedel, T.E. et al. (2012). «Methodology of metal criticality determination». Environmental Science \& Technology, 46, pp. 1063-1070.

Gudynas, E. (2017). «Neo-extractivismo y crisis civilizatoria». En Ortega, G. (coord.), América Latina: avanzando hacia la construcción de alternativas, Asunción (pp. 29-54). Paraguay: BASE Investigaciones Sociales.

Guimaraes, P. y Pérez, J.D. (2016) (eds.). Conflitos ambientais na indústria mineira e metalúrgica: passado e presente. Évora-Río de Janeiro: CEICP/CETEM.

Haikola, S. y Anshelm, J. (2016). «Swedish mineral policy at a crossroads? Critical reflections on the challenges with expanding Sweden's mining sector». The Extractive Industries and Society, 3(2), pp. 508-516.

Hamor, T. (2004). «Sustainable mining in the European Union: the legislative aspect». Environmental Management, 33(2), pp. 252-261.

Haumann, S. (2018). «Towards a historical understanding of critical raw materials». Gaia, 27(4), pp. 373-378.

Humphreys, D. (2010). «The great metals boom: a retrospective». Resources Policy, 35, pp. 1-13.

Instituto Geológico y Minero de España (IGME) (2017). Panorama Minero 2017. Recuperado de http://www.igme.es/PanoramaMinero/PMLin.htm 
Klein, N. (2007). The shock doctrine: the rise of disaster capitalism. Londres: Penguin Books.

Koven, P. (primero de junio de 2012). «How Europe is mining's emerging market». Financial Post.

Landén, A.S. y Fotaki, M. (2018). «Gender and struggles for equality in mining resistance movements: performing critique against neoliberal capitalism in Sweden and Greece». Social Inclusion, 6(4), pp. 25-3.

Lesser, P. et al. (2016). Sustainable mining in the Northernmost Europe. Rovaniemi: Lapin yliopisto.

Mancini, L., Benini, L. y Sala, S. (2018). «Characterization of raw materials based on supply risk indicators for Europe». The International Journal of Life Cycle Assessment, 23, pp. 726-738.

Marinescu, M., Kriz, A. y Tiess, G. (2013). «The necessity to elaborate minerals policies exemplified by Romania». Resource Policy, 38, pp. 416-426.

Nurmi, P.A. y Molnár, F. (2014). «The future of metal minerals mining in the EU». European Geologist, 37, pp. 12-19.

Pérez, J.D. (2014). Tierra devastada. Historia de la contaminación minera. Madrid: Síntesis.

Ramos, A. (s/f). Discrepancia científica con los informes del Instituto Geológico y minero sobre la masa de agua subterránea 05-49 subunidad Gerena-Cantillana [Informe no publicado].

Rayner, J. y Howlett, M. (2009). «Introduction: understanding integrated policy strategies and their evolution». Policy and Society, 28, pp. 99-109.

Šolar, S.V. y Shields, D. (2006). «The effect of policy choices on mineral availability». Geologija, 49(1), pp. 163-172. 
Sonnemann, G. et al. (2015). «From a critical review to a conceptual framework for integrating the criticality of resources into life cycle sustainability assessment». Journal of Cleaner Production, 94, pp. 20-34.

Stefănescu, L., Robu, B.M. y Ozonu, A. (2013). «Integrated approach of environmental impact and risk assessment of Rosia Montana Mining Area, Romania». Environment Science and Pollution Research, 20, pp. 7719-7727.

Tiess, G. (2010). «Minerals policy in Europe: some recent developments». Resource Policy, 35, pp. 190-198.

Velegrakis, G. (2016). «Tales of dispossession in times of crisis: lessons from Greece». En Beltrán, M.J. et al. (eds.), Political ecology for civil society (pp. 75-81). Barcelona: ICTA.

Velicu, I. y Kaika, M. (2015). «Undoing environmental justice: re-imagining equality in the Rosia Montana anti-mining movement». Geoforum, 84, pp. 305-315. 